\section{An adult blind man presenting severe impairment of the right finger, ecchymosis in the thorax region, and haemorrhagic blisters on the oral mucosa}

\section{Editor}

Thrombocytopenia is defined as a reduced platelet count within blood $\left(<100 \times 10^{9}\right.$ units/L), ${ }^{1}$ generating a primary haemostatic defect. $^{2}$ This condition may be observed in diseases associated with (i) impaired platelet production (e.g. aplastic anaemia, myelodysplastic syndrome, chemotherapy), and (ii) increased splenic sequestration (e.g. splenomegaly, disseminated intravascular coagulation, thrombotic microangiopathy). ${ }^{1,2}$ Immune thrombocytopenic purpura (ITP) consists of an acquired immune-mediated disorder with severe dermatological manifestations in which auto-antibodies establish a direct link with specific glycoproteins expressed on platelet surfaces, such as IIb-IIIa and Ib-IX, promoting platelet destruction by macrophages from the reticuloendothelial system. ${ }^{1,3-5}$

A 56-year-old blind man residing in a low-income rural district in the city of Sobral (Brazil), presented to the Stomatology Service of the Federal University of Ceará (Sobral Campus, Brazil) for a dental appointment complaining of oral discomfort. Patient reported a last dental visit 5 years before the present consultation, at the same time in which complete blindness had been diagnosed as a result of uncontrolled systemic hypertension, and untreated glaucoma. The primary care physician had previously screened patient for dietary restrictions, alcohol consumption, drug abuse, hepatitis B/C, HIV infection and diabetes (plasma glucose, $86.9 \mathrm{mg} / \mathrm{dL}$; laboratory reference value, 66$99 \mathrm{mg} / \mathrm{dL}$ ), and patient's medical chart/labs did not confirm the presence of these conditions. Physical examination showed reddened stains and ecchymosis in the thorax region, haemorrhagic petechiae on the fingers, and severe impairment of the right finger (Fig. 1). On intraoral exam, haemorrhagic blisters were observed on the lower lip and left buccal mucosa (Fig. 1), in addition to palatal petechiae and spontaneous gingival bleeding. To investigate any pre-existing blood dyscrasias, a complete haemogram was requested. The haemogram showed a low platelet count of 14000 units $/ \mathrm{mm}^{3}$ (laboratory reference value of 150 000-450 000 units $/ \mathrm{mm}^{3}$ ) and a normal total white blood cell count of $6.1 \times 10^{3} / \mathrm{mm}^{3}$ (laboratory reference value of $\left.4-10 \times 10^{3} / \mathrm{mm}^{3}\right)$. All other laboratory values were within normal limits: red blood cell count (RBC) of $4.32 \times 10^{6} / \mathrm{mm}^{3}$; haemoglobin $(\mathrm{Hgb})$ of $13.4 \mathrm{~g} / \mathrm{dL}$; and a haematocrit $(\mathrm{Hct})$ of $40.3 \%$. In view of these clinical findings, presence of severe thrombocytopenia, and need for dental extractions, the patient was referred to the Regional Center of Hematology and Hemotherapy of Sobral (Brazil) for medical evaluation and appropriate diagnosis. New laboratory exams confirmed the existence of persistent thrombocytopenia (35000 units $/ \mathrm{mm}^{3}$ ), with normal values for $\mathrm{RBC}\left(4.47 \times 10^{6} / \mathrm{mm}^{3}\right)$, $\mathrm{Hgb}(14.6 \mathrm{~g} / \mathrm{dL})$, Hct (42.6\%), mean corpuscular volume (95 fL) and total leucocyte count $\left(6200\right.$ cells $\left./ \mathrm{mm}^{3}\right)$. Thus, the final diagnosis was ITP. ${ }^{6}$ Presently, corticosteroid therapy was the elected treatment alternative (prednisone: $80 \mathrm{mg} / \mathrm{day}$ ), which leads the patient to a gradual regression of oral lesions, re-establishing platelet counts to acceptable levels after a 24-month follow-up period (initial: 14,000 units $/ \mathrm{mm}^{3}$; final: 118,000 units $/ \mathrm{mm}^{3}$ ). In this case, the patient reported interruption and irregular use of the prescribed medication, increasing the severity of thrombocytopenia
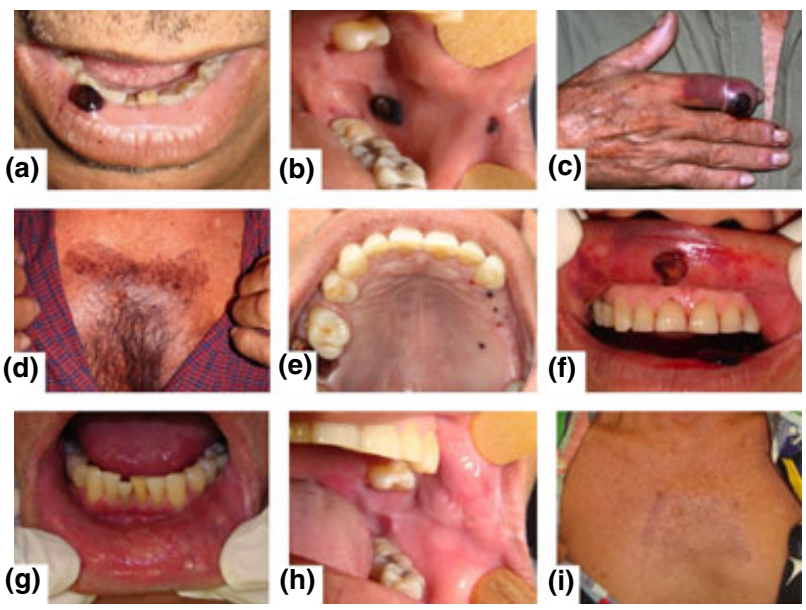

Figure 1 (a) Lower lip showing haemorrhagic blister. (b) Haemorrhagic blisters observed on the left buccal mucosa. (c) Severe impairment of the right finger secondary to thrombocytopenia. (d) Skin lesion on the thorax region. (e) Intraoral view showing partial regression of oral lesions 1 month after the initial examination, with the presence of spontaneous bleeding, petechial lesions, and indication for tooth extraction. (f) Extraoral view of the upper and lower lips at the 18-month follow-up visit showing the presence of haemorrhagic blisters and ecchymosis, demonstrating clinical signs and symptoms of thrombocytopenia. (g) Absence of lip lesions (24month follow-up visit). (h) Normal buccal mucosa. (i) Thorax region showing a normal aspect. 
between the 2nd and 4th follow-up months and a worsening in the oral condition was observed during this period. In brief, this clinical picture highlights the relationship of blood dyscrasias with cutaneous pathologies. In addition, manifestations in the oral cavity may be in association with the first clinical signs of these systemic alterations. Through recognition of dermatological signs and symptoms of ITP coupled with oral mucosal findings, the professional may establish an early diagnosis, and positively interfere in the clinical course of the disease.

F.N. Chaves, ${ }^{1}$ C.S.R. Fonteles, ${ }^{2}$ F.S.R. Carvalho, ${ }^{3}$ T.R. Ribeiro, ${ }^{4}$ K.M.A. Pereira, ${ }^{5}$ F.W.G. Costa ${ }^{4, *}$ ${ }^{1}$ Division of Stomatology, School of Dentistry, Federal University of Ceará, , 'Division of Pediatric Dentistry, Department of Clinical Dentistry, Federal University of Ceará, ${ }^{3}$ Division of Oral and Maxillofacial Surgery, Walter Cantídio University Hospital, Federal University of Ceará, ${ }^{4}$ Division of Clinical Dentistry, School of Dentistry, Federal University of Ceará, Fortaleza, Ceará, ${ }^{5}$ Division of Stomatology, School of Dentistry, Federal University of Ceará Campus Sobral, Sobral, Brazil *Correspondence: F.W.G. Costa. E-mail: fwildson@yahoo.com.br

\section{References}

1 Provan D, Stasi R, Newland AC et al. International consensus report on the investigation and management of primary immune thrombocytopenia. Blood 2010; 115: 168-186.

2 Stasi R. How to approach thrombocytopenia. Hematology Am Soc Hematol Educ Program 2012; 2012: 191-197.

3 Wang L, Li Y, Hou M. Idiopathic thrombocytopenic purpura and dysmegakaryocytopoiesis. Crit Rev Oncol Hematol 2007; 64: 83-89.

4 Mazzucconi MG, Fazi P, Bernasconi S et al. Therapy with high-dose dexamethasone (HD-DXM) in previously untreated patients affected by idiopathic thrombocytopenic purpura: a GIMEMA experience. Blood 2007; 109: 1401-1407.

5 Kosugi S, Tomiyama Y, Honda S et al. Platelet-associated anti-GPIIb-IIIa autoantibodies in chronic immune thrombocytopenic purpura recognizing epitopes close to the ligand-binding site of glycoprotein (GP) IIb. Blood 2001; 98: 1819-1827.

6 Mahabir VK, Ross C, Popovic S et al. A blinded study of bone marrow examinations in patients with primary immune thrombocytopenia. Eur J Haematol 2013; 90: 121-126.

DOI: $10.1111 / j d v .12460$ 